Щ्)FRANÇAISE

$\supset$ DE

필 PÉDAGOGIE

\section{Revue française de pédagogie}

Recherches en éducation

154 | janvier-mars 2006

La construction des politiques d'éducation : de nouveaux rapports entre science et politique

\title{
Quand le courant pédagogique « des compétences » empêche une structuration des enseignements autour de l'étude et de la classification de questions parentes
}

When educational trends regarding "learning skills" prevent the structuring of teaching methods based on studying and classifying main issues

Wenn die pädagogische auf den Kompetenzen basierende Strömung einer

Strukturierung des Unterrichtens um die Bearbeitung und die Klassifizierung verwandter Fragen im Wege steht

Cuando la corriente pedagógica « de las competencias » impide una estructuración de las docencias alrededor del estudio y de la clasificación de cuestiones semejantes

\section{Maggy Schneider-Gilot}

\section{OpenEdition Journals}

\section{Édition électronique}

URL : http://journals.openedition.org/rfp/136

DOI : $10.4000 /$ rfp. 136

ISSN : 2105-2913

Éditeur

ENS Éditions

Édition imprimée

Date de publication : 1 mars 2006

Pagination : 85-96

ISBN : 2-7342-1039

ISSN : 0556-7807

Référence électronique

Maggy Schneider-Gilot, « Quand le courant pédagogique « des compétences » empêche une structuration des enseignements autour de l'étude et de la classification de questions parentes », Revue française de pédagogie [En ligne], 154 I janvier-mars 2006, mis en ligne le 01 mars 2010, consulté le 10 décembre 2020. URL : http://journals.openedition.org/rfp/136; DOI : https://doi.org/10.4000/rfp. 136 


\title{
Quand le co urant pédagogique “deS Compéten CeS» empêChe Une structuration deS enSeignements auto ur de l'étude et de la classific don de questions parentes
}

\author{
Maggy Schneider-Gilot
}

Cet article montre et analyse un phénomène lié à la mouvance dite "des CompétenCes": en déplaçant l'ac Gnt sur des CompétenCes Communes à plusieurs disCiplines, le mythe du transfert Conduit à un éCrasement des spéCifiCités épistémologiques et, par là, à un phénomène de "déC ágorisation" des questions étudiées à l'éCole. Les faits analysés relèvent de plusieurs disciplines (prinCipalement, les $m$ athématiques, l'histoire et les sCienCes), ansi que de l'interdisCiplinarité. Ils ont été observés en Belgique $m$ ais sont signific afs de phénomènes qui existent ailleurs.

De Scripte urs (TEE) : apprentissage, Compétence, mathématiques, savoir, transposition didactique

C: que l'on appelle désorm ais le courant pédagogique des compétences - et que d'aucuns n'hésitent pas à nommer la doxa des Compétences (Crahay, 2006) - influence désormais les programmes scolaires en de nombreux pays.

Cette mouvance pédagogique et son origine dans le monde professionnel ont fait l'objet de nombreuses études dont la bibliographie jointe contient un échantillon assez représentatif je Crois. J'y renvoie le lecteur qui souhaite s'informer plus avant sur les fondements d'un tel Courant et sur les débats que soulève le ConCept même de CompétenCe. Mon but n'est pas d'approfondir la question de Ce point de vue-là. Je me placerai plutôt au niveau du terrain, ayant pu y observer quelques pratiques ensei- gnantes, liées tantôt aux mathématiques, tantôt à d'autres disciplines, qui se réclament de Ce Courant pédagogique en référence explicite ou non au Concept de transfert et j'analyserai Ces pratiques Comme des faits divers révélateurs d'une Certaine "décatégorisation" des questions étudiées à l'école et d'une forme de négation des spéCifiCités disCiplinaires. Je terminerai en pointant, par quelques exemples, le type de réflexion épistémologique dont il Conviendrait de tenir Compte, discipline par dis Cipline, pour restaurer à chacune ce qui la rend non réductible aux autres.

Comme l'illustre P. Jonnaert (2003), les diverses acceptions du mot Compétence renvoient tantôt à des capacités formelles (telle que la résolution de 
problèmes), tantôt à des compétences plus spécifiques s'exerçant sur des objets de savoir dont on tente d'identifier les $C$ aactères épistémologiques. Ainsi, Comme le rem arque Cet auteur, les "éléments que le sujet peut mobiliser pour traiter une situation avec suc 6s" vont, selon les CherCheurs, de "Comportements" (Raynal \& Runier, 1997) à un "système de ConnaissanCes ConCeptuelles et proCédurales " (Gillet, 1991), en passant par des "dispositions de nature Cognitive, affective, réflexive et Contextuelle" (Pallascio, 2000). On peut penser que les dérives dénoncées dans Cet article ConCernent une interprétation des Compétences en termes de c a acités formelles. Et pourtant... Les exemples relevés iCi sont à interpréter, pour la plupart, dans Ia perspective d'un décret (Belgique, 1997) qui définit les missions prioritaires de l'enseignement belge francophone en introduisant "notamment une pédagogie plus active et Centrée sur les Compétences", ainsi que le Commentent H. Draelants et al. (2003). Or, ce déCret est loin de négliger les Compétences plus spéCifiques puisqu'il définit le concept de Compétence Comme une "aptitude à mettre en $œ u v r e$ un ensemble organisé de savoirs, de savoirfaire et d'attitudes permettant d'accomplir un Certain nombre de tâches... ».

Si les faits analysés dans cet article ont été observés essentiellement en Belgique, ils sont significatifs, je pense, d'une tendance qui se manifeste ailleurs, même si, dans ce pays, une forte sensibilité actuelle aux problèmes d'évaluation $a \mathrm{pu}$ amplifier le phénomène relaté. Je pense, par exemple, aux textes officiels qui, au Québec, exposent les traits saillants de la formation scolaire (Québec, 2001). J'y reviendrai pour faire un rapprochement avec Ia perspective belge.

\section{DES PRATIQUES SOUS L'ÉGIDE DES COM PÉTENCES QUI ÉVITENT TOUTE CATÉGORISATION DES QUESTIONS ÉTUDIÉES PAR LES ÉLÈVES}

Pour étayer l'opinion résumée dans Ce titre, je suis partie principalement de documents reconnus dans Ia noosphère Comme représentatifs de ladite réforme des Compétences. Il s'agit globalement, m ais Ce sera préCisé à chaque fois, soit de doCuments rédigés $p a r$ des Commissions officielles Chargées de rédiger des programmes ou des outils d'évaluation, soit de recherches Comm anditées par Ia Communauté française de Belgique à l'occasion de Cette réforme. Mes exemples porteront sur quelques disciplines seule$m$ ent $m$ ais le caractère représentatif $d e$ Ceux-Ci a été Corroboré par une lecture plus vaste des pro. grammes scolaires, toutes disCiplines Confondues.

Il s'agit toutefois d'une investigation portant prinCipalement sur l'enseignement seCondaire mais il n'est pas difficile de trouver des tendances équivalentes dans l'enseignement primaire ou, plus récem. ment, dans l'enseignement universitaire. Je me suis également référée à des observations faites dans des classes et significatives des phénomènes analysés iCi.

\section{La ré So Iution de p roblè $m$ e $S$ et Son é valuation Considé rées Com m e Un absolu}

Le premier fait divers qui sera déCrit ConCerne Ce que l'on appelle les "problèmes de dénombrement" au niveau des deux premières années de l'enseignement secondaire, dans les programmes scolaires belges ou dans les manuels. Ces problèmes, présents également dans les enquêtes PISA, supposent d'identifier d'abord une régularité dans une suite de nombres figurés par des objets et d'exprimer ensuite Cette régularité par une formule susceptible de donner le nombre d'objets à toute étape. Des profes. seurs enseignant dans des écoles voisines font part à un Conseiller pédagogique de leur perplexité face à Ces problèmes que leurs élèves échouent souvent à résoudre. Le Conseiller rassemble plusieurs de Ces problèmes et explique aux professeurs, qui n'en sont $p$ as Conscients, les diverses structures fonctionnelles sous-jacentes à Ces problèmes. Dans le cas présent, Celles-Ci sont au nombre de Cinq et des techniques particulières permettent de les repérer et de les traiter au-delà du Contexte : ainsi, une suite arithmétique se traduit $p$ ar des écarts Constants d'un terme au suivant et la formule associée ne fait que rendre Compte du nombre de fois qu'il faut ajouter Cet écart pour obtenir un terme donné... Les professeurs apprécient l'enseignement prodigué par le Conseiller pédagogique car, disent-ils, Ce discours les aide eux-mêmes à y voir plus clair dans le "fouillis" des problèmes qu'ils parviennent enfin à "catégoriser". Le conseiller pédagogique les engage à travailler ces problèmes de la m ême façon avec leurs élèves. La réponse des professeurs est le fait que je voudrais analyser iCi: "Si nous faisons Ia $m$ ême Chose avec nos élèves, Ce ne sera plus pour eux de la résolution de problèmes".

Cette réaction caractérise un Certain usage pédagogique des problèmes de dénombrement : leur 
portée est perçue avant tout Comme oc Csan d'entraînement des élèves à la résolution de problèmes. Mais chacun de ces problèmes doit se résoudre presque indépendamment des autres, le progrès ne pouvant venir que de l'exercice même de la démarche de résolution de problèmes. Il n'y $a$ iCi auCune "C aégorisation"de Ces problèmes. Celle-Ci est pourtant envisageable et pourrait inspirer un enseignement où le classement des problèmes de dénombrement aurait autant de place que leur résolution, enseignement qui se solderait par l'institutionnalisation (au sens de Brousseau, 1998) des structures fonctionnelles mobilisées par les problèmes étudiés et des techniques qui permettent de les identifier.

La perspective pédagogique dans laquelle Cette $m$ anière d'envisager les problèmes de dénombrement prend tout son sens semble bien être Celle d'un enseignement axé sur le développement de la "résolution de problèmes", dém $\operatorname{arche~qui,~de~tout~}$ temps, a été Considérée Comme importante en m athématiques et dont la mouvance des Compétences redore le blason au point d'en faire un objectif important dans d'autres disciplines. Cette perspective est Celle qui sous-tend des recherches Comm anditées par la Communauté française de Belgique et que le travail de J.-P. Cazzaro et al. (2001) illustre parfaitement. Je me Contenterai de résumer iCi une analyse plus détaillée de Cette recherChe (Schneider, 2002a). L'intention déclarée de ses auteurs est de "structurer l'enseignement des $m$ athém atiques par des problèmes ", par le biais soit $d e$ problèmes d'introduction, soit de problèmes d'application. Bien sûr, dans Cet ouvrage, les problèmes du premier type sont Censés déboucher sur un pan de théorie mathématique même si, en l'absence d'analyse épistémologique et didactique, l'articulation entre activités des élèves et théorisation n'y relève pas forCément d'une "néCessité " intrinsèque au dispositif didactique imaginé. Mais, Ce qui est à souligner iCi, C'est le fait que les auteurs Considèrent surtout les problèmes, tant les problèmes d'introduction que les autres, comme matière à évaluation formative de la Compétence "résolution de problèmes " alors qu'un apport en termes de savoirs pourrait être déclaré l'objectif premier. De manière très significative, ils empruntent à $A$. Sfard (1991) les trois phases qu'elle distingue dans la formation d'un ConCept : intériorisation, Condensation et réification non seulement pour Construire des séquences d'enseignement mais aussi pour modéliser la dém arChe de résolution de problème et pour structurer des grilles d'évaluation de Cette Compétence. Ils détournent ainsi une grille de lecture de sa fonCtion pre mière pour l'interpréter à la lumière de l'idéologie des Compétences. Plus généralement, l'accent est $m$ is, dans Cette recherche, sur les aspects méthodologiques de la résolution de problèmes, à la m anière de G. Polya (1967) qui étudie des stratégies telles que l'étude de cas particuliers, et non sur un quelConque Classement des problèmes: hormis dans Ia description locale de Certaines séquences d'enseignement, les problèmes détaillés dans l'ouvrage n'y sont catégorisés que par le biais de démarches telles que "géométriser" et "numériser", démarches transversales dans la mesure où elles peuvent être a priori fécondes dans tous les problèmes mathém atiques quelle que soit la question traitée.

Les deux exemples préCédents sont révélateurs, d'une part, d'une Conception de la résolution de problèmes qui relève d'une certaine quête d'absolu (Comme déCrit dans Schneider, 2002b) et, d'autre part, d'une définition très générale de Ce qu'est un problème, généralement non questionnée, dans Iaquelle on trouve des références au caractère inédit et/ou Complexe : "Un problème est une question suffisamment Complexe pour que la réponse ne soit pas facile à trouver" (Cazzaro et al., 2001). Cette dernière définition est assortie de caractéristiques très diversifiées allant de la difficulté à résoudre le problème à son impact sur la motivation des élèves. Comme C'est le cas Chez Ces auteurs, Cette ConCeption de la résolution de problèmes s'accommode $m$ al d'une catégorisation des problèmes surtout si Celle$\mathrm{Ci}$ est faite sur base de Critères liés à leur résolution. Et C'est bien Ce qu'expriment les professeurs rétiCents à montrer à leurs élèves les structures fonctionnelles sous-jacentes aux problèmes de dénom. brement sans doute dans la crainte de voir les problèmes se Convertir en exerCices "répétitifs" et leur résolution en "reCettes". Pour eux, Ia résolution de problèmes est ou n'est pas et tout enseignement pollue en quelque sorte la pureté de la démarche. Sans doute, le spectre d'une évaluation "scientifique ", souvent associé à la réforme des Compétences, participe à cet absolutisme. Une telle évaluation est Censée "mesurer" l'objeCtif annonCé. Or, disent les enseignants, Comment évaluer rigoureusement la progression des élèves en $m$ atière de résolution de problèmes si Ce n'est en leur soumettant de "vrais" problèmes à résoudre, C'est-à-dire des problèmes qu'ils n'ont pas encore rencontrés? Toute entreprise susceptible d'aider les élèves dans 
Cette tâche ne peut qu'interférer avec Glle d'une évaluation scientifique de la dém arche visée et C'est Ià un paradoxe sur lequel je reviendrai plus loin.

\section{De $\mathbf{S}$ m atrice $\mathbf{S}$ d'é Valuation in te rche angeable $\mathbf{S}$}

Comme les exemples préCédents le suggèrent, C'est par le biais de l'évaluation que la mouvance des Compétences pénètre les pratiques enseignantes en Belgique. Dans la foulée du déCret "Missions" est prévue la Construction de "référents" externes en matière d'évaluation des Compétences. Des Commissions ad hoC sont Chargées de rédiger des situations d'évaluation. Celles qui travaillent sur les Compétences terminales, au niveau du lyCée, le font suivant le modèle de J. Beckers (2002) dans lequel la notion de "famille de tâches" est Centrale et définit ce que l'on a encore appelé "matrice d'évaluation ". Le ConCept de tâche est pris iCi au sens des ergonomes tels J. Leplat (1992), C'est-àdire qu'une tâche est définie par le but à atteindre (les finalités) et par les conditions dans lesquelles il doit être atteint. J. Beckers (2002) ajoute que "la tâche [...] sera Complexe au sens où elle doit potentiellement déséquilibrer les élèves, être sourCe de tension pour eux, et donc ne pas leur être familière ". Quant à l'idée de famille, elle suggère que plusieurs tâches puissent avoir une parenté telle que le travail fait sur l'une d'elles favorise l'exécution d'une autre tâche de la même famille. J'y reviendrai.

Je voudrais illustrer iCi Comment se définit Cette parenté des tâches d'une même famille, parenté qui pourrait Constituer a priori une Certaine forme de catégorisation. Examinons, de Ce point de vue, trois matrices d'évaluation en sciences. Elles sont extraites d'un travail en Cours (dans le courant de l'année 2003 ) et l'analyse faite iCi ne préjuge auCunement de la suite qui y a été donnée ni de l'évolution du travail des Commissions ConCernées. Ces matrices sont Cependant significatives, à un moment donné, d'une tendance à écraser des spéCifiCités disciplinaires derrière des formulations très transversales. La prem ière " propose à l'élève de s'approprier des notions de physique, de résoudre des problèmes et de Com muniquer les résultats qu'il a obtenus". La seconde Concerne la chimie et "propose à l'élève de résoudre des applications Concrètes et d'utiliser des savoirs scientifiques pour enrichir des représentations interdisCiplinaires". La troisième "propose aux élèves de répondre par éCrit à une question relative à ıa biologie en s'appuyant sur des théories scientifiques". Le fait majeur que je tiens à souligner ici est que la formulation de ces familles de tâches est épistémologiquement neutre. En effet, on peut éChanger les disCiplines sCientifiques dans Chacune de Ces matrices sans perdre ni sens, ni intérêt d'ailleurs. En revanche, Cette interchangeabilité interdit de situer la matrice dans une discipline scientifique donnée si Celle-Ci n'est pas mentionnée explicitement et a fortioride la situer dans un ou plusieurs domaines précis de Cette discipline : mécanique, éleCtrocinétique, équilibres Chimiques, immunologie... En outre, Ces matrices ne permettent en aucune manière de distinguer a priori plusieurs caté. gories de problèmes au sein de Chacune de Ces disCiplines ou sous-disciplines Ainsi, Celle relative à la biologie est illustrée par l'explicitation de dysfonctionnements du système immunitaire m ais elle aurait pu tout aussi bien recouvrir des problèmes de clas. sification des êtres vivants. Il semblerait donc bien que la Constitution de matrices d'évaluation soit, au départ, relativement étrangère à une quelconque catégorisation des questions étudiées au sein même des sciences et qu'une éventuelle parenté entre les diverses tâches d'une même famille ne soit pas à Chercher, dans ces documents, au niveau de l'épistémologie propre des disCiplines ConCernées et de leurs sous-disciplines $m$ ais bien à Celui des Compétences transversales exercées ou encore, Comme entendu lors de formations et illustré par la matrice de biologie, au niveau d'aspects assez formels de la production attendue, par exemple, le fait de demander un texte écrit ou un exposé oral.

Mon troisième exemple est le référentiel d'histoire lequel illustre également l'accent $m$ is sur des aspects transversaux par rapport aux disciplines. Te que déCrit par J. Beckers (2002) Ce référentiel disCerne quatre Compétences : se poserdes questions, Critiquer, synthétiser et Communiquer, articulées à des familles de tâches voire identifiées à elles. La première Compétence Couvre deux familles de tâches : "se poser des questions" et "se documenter sur une question". La deuxième Compétence reprend Cette dernière tâche et Com. prend en outre la famille: "analyser et Critiquer des sources". La Compétence synthétiser est spéCifiée par la famille "Établir une synthèse et formuler une hypothèse explicative". Quant à la Compétence Communiquer, elle est préCisée par la famille "Communiquer un savoir historique". Les Conditions de réalisation de la tâche sont préCisées, en partiCulier, les types de documents disponibles : iConographies, textes... Enfin, Ces CompétenCes doivent être Croisées avec des Concepts et des moments Clefs dont le Choix est déclaré arbitraire pourvu 
"qu'ils aient été travaillés en classe". Dans Ce référentiel, la seule mention qui est faite à la discipline Concernée est englobée dans la dernière famille: C'est bien un savoir historique qu'il s'agit de Communiquer. Et l'on im agine aisément une grille de Compétences analogue pour la géographie avec des supports et Concepts différents, du moins en partie.

Voici un exemple de situation d'évaluation imaginée à partir du référentiel qui vient d'être déCrit (Cecafoc, 2003). Il s'ag it de clarifier la situation dans les Balkans à ıa veille de la première guerre mondiale. Des documents sont fournis : cartes et déclarations politiques entre autres. Les élèves doivent produire une synthèse de deux pages dans laquelle ils identifient les différents protagonistes et interprètent le type de tensions en présence. L'outil interprétatif est le concept de nationalisme étudié auparavant en classe à propos des tensions européennes de la fin $d u$ XIXe siècle et $d u$ début $d u x^{e}{ }^{e}$ siècle. Parmi les Critères d'évaluation relatifs au fond, les auteurs prévoient que "les motifs des tensions soient identifiés à la lumière d'un Concept" sans préCiser lequel. Cependant, plusieurs des doCuments fournis (tracts et déclarations politiques) induisent l'exploitation exclusive du Concept de nationalisme, Ce qui est attendu effectivement de l'élève, alors que d'autres concepts sont susceptibles a priori d'éclairer un Conflit, tel que le ConCept de Crise qui prend en Compte une dimension éConomique. La Compétence qui labellise le travail dem andé est "synthétiser". Sa formulation m asque Complètement le savoir mobilisé. De plus, il ne s'agit nullement d'une Compétence dont l'exerCice est le monopole de l'histoire. À nouveau, on ne voit se profiler aucune spéCification ni catégorisation des questions propres à Cette discipline dans l'énonCé même de la Compétence alors que l'exemple donné Cidessus pourrait suggérer l'énoncé de questions types étudiées au Cours d'histoire telles que "analyser un Conflit". D'autres catégories de questions peuvent être imaginées, par exemple "situer un événement dans son époque ", questions dont on pourrait imaginer une classification en Croisant deux Critères : l'objet même de la question et les Concepts qu'elle mobilise parmi Ceux répertoriés: migration, Croissance, démocratie... Le programme HistoireFormation historique, $2^{e}$ et $3^{e}$ degré édité par la Fédération de l'enseignement secondaire catholique (FESeC) en 2000 , inspiré du référentiel supra, fait un $p$ as dans ce sens. En effet, ses auteurs y voient le Concept Comme "une Clé de lecture qui doit permettre au jeune de faire face à des situations nouvelles" car "forgé par la Confrontation de situations divergentes et la $m$ ise en lumière des invariants qui les rapprochent". Cependant, Ce programme et l'évaluation associée sont globalement articulés autour des compétences répertoriées plus haut. Quant au Choix et à l'ordonnancement des Concepts, ils sont peu questionnés dans le programme, leur caractère Contraignant se justifiant surtout par la néCessité de préCiser les pré-requis sur lesquels le professeur peut tabler d'une année à l'autre. Tout Cela occulte la possibilité d'un Cours d'histoire organisé à $p$ artir de questions-types, à l'instar d'un Cours Conçu par A. Maingain (2000) où les élèves sont invités à regrouper, différencier et reconnaître des situations relevant de divers systèmes idéologiCo-politiques à la lumière des outils que sont les Concepts tels que le totalitarisme, la social-démocratie, l'impérialisme... Dans un tel cours, le Choix du Concept "analyseur» est, à partir d'un moment donné, à charge de l'élève Contrairement à la situation décrite au début de ce paragraphe. Et seul un travail préalable d'analyse des invariants et des spéCifiCités, Conduisant à une catégorisation des situations rencontrées, peut autoriser une telle dévolution.

D'une m anière générale, on observe une nette tendance à définir les familles de tâches à partir de "démarches" Communes à plusieurs disciplines. Ainsi, en Ce qui ConCerne les socles de CompétenCes à l'issue des deux premières années du secondaire, B. Rey et al. (2002) observent que l'élém ent Commun à la famille tient parfois, soit à une démarche mentale telle que "traiter l'information " en français, soit à une "méthodologie qui est une garantie de scientificité". Ainsi les Compétences visées en form ation historique et géographique, tout Comme Celles exerCées en éveil scientifique relèvent de méthodologies de recherche somme toutes assez proches allant de l'observation ou de la recherche de l'inform ation à la Communication d'une Conclusion. Ce qui n'est pas sans rappeler la fascination exercée par la dém arche OHERIC (Observation, hypothèse, expérimentation, résultats, interprétation, Conclusion), par ailleurs tant déCriée par les épistémologues des sciences pour son hypothèse implicite d'une possible observation neutre et objective (Fourez, 2003). Quant aux $m$ atrices d'évaluation relatives aux CompétenCes ter$m$ inales en mathématiques, il semble bien qu'elles doivent être obligatoirement structurées, par une sorte de dictat pédagogique, autour de "Critères" (sic!) qui rappellent les différentes étapes de la démarche de résolution de problèmes: Examen et traduction des données, modélisation $m$ athém atique, 
traitement de la modélisation et rédaction de la solution $d u$ problème et dont l'adaptation à différents problèmes issus tant de la géométrie que de l'algèbre semble soulever bien des difficultés.

Cette insistance vive sur les dém arChes générales ne sont pas propres, ni au Contexte de l'évaluation, $\mathrm{ni}$ à l'enseignement belge. Ainsi, le ministère de l'Éducation de Québec (Québec, 2001) les met à l'avant-pıan pour présenter les traits saillants de ses programmes scolaires en général et de Celui relatif aux m athém atiques en particulier. Ce dernier s'organise en effet autour des trois Compétences: résoudre une situation-problème, déployer un raisonnement mathém atique et Communiquer à l'aide $d u$ langage $m$ athém atique. On rem arquera que Ia spécificité disciplinaire se marque par l'usage du qualificatif “mathématique", mais que le remplacement de Celui-Ci par le qualificatif "sCientifique " pourrait bien suffire à préCiser la teneur d'un programme de sciences.

\section{Une vSion uhilaté rale de l'in te rdiscip linarité}

Le dernier exemple analysé iCi a trait à l'interdis Ciplinarité telle qu'envisagée par A. Maingain et al., 2002. Il s'agit principalement d'exploiter une méthodologie de Construction d'un îlot de rationalité vue Comme "une pratique intégratrice de savoirs issus de diverses disciplines en vue d'approcher les problèmes dans leur particularité " [je souligne]. Cette méthodologie est largement inspirée de grilles de lecture sociologiques et Consiste à :

- problématiser la démarche en posant la question "De quoi s'agit-il? " et préCiser le projet: Contextes, finalités, destinataires, produit ;

- faire émerger le Cliché : Ce dont on tient Compte spontanément ;

- établir le panorama : ce dont on pourrait tenir Compte : acteurs, Contraintes, enjeux, tensions et Controverses, sCénarios envisageables ;

- Clôturer la démarChe : sélectionner les aspeCts dont on tiendra compte, les boites noires à ouvrir, hiérarchiser les données.

Les points d'ancrage de tels travaux interdisciplinaires proposés dans l'ouvrage sont très diversifiés: une problématique, une situation de vie, la réalisation d'un projet tel qu'une exposition, un débat, une donnée de l'actualité, Ia préparation d'un voyage, ıa $m$ ise en place de Compétences transversales... $E_{t}$ les exemples inspirés par ce travail ne le sont pas moins : Cela va du Choix d'orientation après un CyCle d'études à l'usage du téléphone mobile dans la vie quotidienne en passant par la solarisation d'un village africain.

Même si les auteurs insistent sur la Complémentarité entre approche disciplinaire et approche interdisciplinaire, ils inscrivent résolument leur modèle dans une dialeCtique du disciplinaire et $d u$ non disCiplinaire. Leur but affiché Consiste effeCtivement à adopter une approche systémique, "un des paradigmes Cognitifs dominants dans Certains secteurs de la recherche et dans Certains lieux de déCision", pour pallier les limites du Compartimentage des savoirs réalisé par l'approche disciplinaire. Leur philosophie est de développer une pratique essentiellement "politique" qui suppose de négoCier et de déCider la part des diverses disciplines impliquées pour réaliser un projet particulier.

Cette vision de l'interdisCiplinarité est Cependant assez unilatérale en Ce sens qu'elle néglige l'intérêt qu'il y aurait à rapprocher des problèmes interdis Ciplinaires parents Comme Ceux liés aux biens de Consommation ou les problèmes de santé afin de dégager des invariants et des spéCifiCités dans leurtraitement. Une telle approche Conduit d'ailleurs, dans un Certain nombre de cas, à la constitution de nouvelles disciplines à l'intersection de disciplines déjà existantes : Ia biochimie et l'informatique en sont des illustrations. La pratique de l'interdiscipli$n$ arité développée par A. Maingain et al. semble même s'être Construite en opposition à Cette autre perspective d'interdisCiplinarité que l'un des auteurs mentionne dans un ouvrage antérieur (Fourez, 1988) pour la Critiquer Comme induisant l'illusion d'une "superscience". Cette position des auteurs relève ainsi d'un choix non questionnable qui apparaît Comme tel lorsqu'on la confronte à d'autres approches de type interdis Ciplinaire.

En outre, la variété des points d'ancrage des travaux interdisciplinaires, souhaitée par les auteurs, n'est pas forCément Compatible avec la néCessité, soulignée par Y. Chevallard et Y. Matheron (2002), d'axer de tels travaux surdes questions susceptibles d'être problématisées par les élèves eux-mêmes et dont la problématisation suppose une réelle Codisciplinarité. Et, Comme l'ont analysé plusieurs Chercheurs en didactique lors d'une réCente éCole d'été (Matheron \& Schneider, 2005), il n'existe à I'heure actuelle aucun cadrage didactique satisfaisant de l'interdisciplinarité permettant d'éviter, autant que faire se peut, les réalisations du terrain 
qui relèvent du "reCopiage Culturel" (Chevallard \& Matheron, 2002) sans mobiliser vraiment ni une réelle problématisation de la question $m$ ise à l'étude, ni une "Co-disciplinarité" de la part des professeurs Concernés lesquels préfèrent souvent laisser aux Collègues le soin "d’éclairCir" la boîte noire disciplinaire qui ne les Concerne pas. En particulier, si l'on veut apprendre aux élèves à problématiser des questions socialement vives - et sans doute est-Ce là la principale finalité de l'interdis Ciplinarité -, peutêtre faut-il leur apprendre à catégoriser ces questions au-delà de l'usage des méthodologies générales existantes qui, malgré leur intérêt épistémologique, ne peuvent avoir une efficacité universelle en regard de tous les problèmes d'interdis $\mathrm{Ci}_{-}$ plin arité.

Plusieurs des pratiques déCrites Ci-dessus font fi d'une quelconque efficacité à "Catégoriser" les problèmes. En mettant l'accent sur Ce qui fait l'essence de la dém arche de résolution de problèmes $m$ athém atiques, tous problèmes Confondus, on évite de Classer Ceux-Ci selon l'un ou l'autre Critère : Ia structure fonCtionnelle mobilisée dans les problèmes de dénombrement par exemple ou encore la nature de la question étudiée : Construction d'un objet géométrique ou problème d'optimisation. Pourtant, les $m$ athém atiques se divisent en rubriques qui rassemblent des problèmes proches en Ce sens qu'ils se résolvent $a u$ moyen de mêmes techniques: l'algèbre linéaire élémentaire, la géométrie des transformations...

De même, les matrices d'évaluation en sciences ou en histoire analysées plus haut ont, telles que formulées, un caractère presque interchangeable. On n'y voit pas apparaître un quelconque classement de problèmes proches selon l'un ou l'autre Critère.

Quant à l'exemple relatif à l'interdisciplinarité, il montre une volonté de traiter chaque problème dans sa particularité, sans souci de le ramener à un éventuel problème parent.

\section{LE MYTHE DU TRANSFERT}

\section{L'éVitem ent d'Une que IConque catégorisation qui Se justifie par ia Vo ıonté de favorise $r$ le $S$ tran Sfe rts}

Mais d'où vient Cet évitement presque délibéré d'une Certaine catégorisation des questions étudiées à l'école? C'est là, je pense, que joue la réfé- rence, éventuellement implicite, au phénomène dit de transfert. Comme le fait remarquer P. Perrenoud (2002), le Concept même de transfert est ambigu. Derrière Ce mot se cachent en effet, des réalités diverses: le transfert d'une technique apprise pour résoudre un problème nouveau au sein d'un même Cours, un transfert de Concept d'une discipline à l'autre, des Compétences méthodologiques et j'y verrais aussi des postures telles que l'intention rationnelle de B. Rey (1996). Il n'empêche que Ce Concept de transfert semble Cristalliser toutes les attentes vis-à-vis de l'éCole, d'autant plus vives que Cette dernière est en Crise. Ainsi, B. Rey (1996) montre en quoile Concept de Compétence transversale, sous-tendu en filigrane par Celui de transfert, relève d'un mythe fondateur de l'école au point de devenir, à l'heure actuelle, "vecteur d'injonction institutionnelle", mythe qu'il déCrit en Ces termes significatifs : "L'idée de Compétence transversale n'est qu'une idée de pédagogue ou de didacticien qui souhaite optimiser les effets de l'enseignement et qui voudrait que les acquis des élèves s'étendent bien au-delà de leur domaine d'apprentissage". J. Tardif (1999) pointe le même phénomène en utilisant l'expression significative de "pierre philosophale du transfert "

Le fait d'affirmer que les apprentissages scolaires ont des visées qui les transcendent ne me Choque pas. Mais, Comme S. Johsua (2002), je Crains que Cet engouement aveugle pour le transversal ne se solde par une sorte d'évitement des moyens a priori Crédibles et adaptés à une telle visée : "Avec la forte influence de "l'entrée par les Compétences", nous serions finalement en présence d'un véritable détournement de problématique, en même temps que d'une dérobade (Comme on dit d'un Cheval qu'il se dérobe devant l'obstacle). Le questionnement initial est tout à fait recevable, indispensable même : quels sont exactement les liens entre les apprentissages formels et Ceux qui se produisent "en situation"? Comme on ne sait pas bien répondre à Cette question, et qu'il est Courant de Considérer que les premiers sont tout sauf "utiles", Ia tentation est grande de se limiter aux seconds. Mais, en toute rigueur, si Ce choix était fait, C'est toute l'institution scolaire qui serait obsolète, puisque, par définition, les savoirs acquis "en situation"nécessitent justement d'être "en situation". On Cherche alors à fonder la spéCificité scolaire sur l'abord de Compétences de niveau supérieur, "générales", dont aucune base théorique ne parvient pourtant à fonder la pertinence de manière Convaincante. C'est le détournement de la problém atique." 
Il me semble que l'évitement d'une C ágorisation des questions abordées à l'éCole relève d'un même processus de "dérob ae ": dans la mesure où l'on Cherche à favoriser les transferts d'une situation à l'autre, tout ce qui est Commun à Ces situations ras. sure et donne l'illusion de la faisabilité du transfert, tout Ce qui distingue Ces situations rappelle que tout transfert suppose une adaptation et une gestion des spéCifiCités qui ne vont pas de soi. D'où le suCCès des Compétences "transverses" par rapport à Ces situations fussent-elles ou non issues d'une même dis Cipline.

J'en veux pour preuve la prégnance des références au transfert dans les cas relatés à la section 1. Ainsi, J.-P. Cazzaro et al., 2001 , situent leur démarche, d'entrée de jeu, dans la problém atique du transfert : "Le problème du transfert apparaît Comme crucial car C'est Cette capacité de transfert qui permet à l'individu de s'adapter à des situations nouvelles pour lui ". A. Maingain et al. (2002) présentent leur modèle de l'interdisciplinarité Comme un des deux pôles, l'autre étant la transdisciplinarité, qui déClinent un même paradigme éducatif nouveau : "la formation devrait désormais Connecter les disciplines entre elles pour rendre les apprenants capables d'avoir un "regard liant". I appartient donc aux enseignants de diffuser Ce nouveau Comportement intellectuel». Et C'est dans Ce paradigme qu'ils situent la méthode de Construction d'un îlot de rationalité, méthode qu'ils Choisissent préCisément pour son caractère transférable d'un problème à l'autre. Quant au concept de famille de tâches, il semble bien délimiter un espace à l'intérieur duquel le transfert devrait s'opérer, si l'on en juge par Ce propos de J. Beckers (2002), lourd d'un tel implicite: "Le type d'activité sollicité par la tâche peut-il suffire à distinguer les familles de tâches? La réponse est clairem ent non si la question sous-entend un transfert automatique entre les tâches d'une même famille». En Ce qui concerne l'évaluation de la Compétence "résolution de problèm es ", Cette référen Ce au transfert interdit presque tout enseignement préalable si l'on prend, à l'instar des professeurs Cités plus haut, le caractère transversal de Cette Compétence au pied de la lettre. En effet, Cet enseignement empêcherait vraiment d'observer si les élèves transfèrent spontanément la démarche de résolution de problèmes qui, par sa description vraiment fort générale, Couvre tous les problèmes mathématiques et doit, par définition même, s'exerCer et s'évaluer sur des problèmes nouveaux pour l'élève. Seul l'entraînem ent à la dém $\operatorname{arche}$ générale est permis et il serait d'autant plus efficace, aux dires de Certains de Ces professeurs, que l'on aura proposé aux élèves des problèmes différents d'une fois à l'autre. Et, comme dit plus haut, Cette situation engendre une tension inévitable entre le souci d'une évaluation scientifique et, tout simplement, la volonté d'enseigner.

Cette analyse me pousse à Conclure que C'est effectivement Cet espoir de transférabilité qui explique l'accent mis sur ce qu'il y a de Commun d'un problème à l'autre ou d'une discipline à l'autre. À Cela s'ajoute sans doute, pour Certaines dis Ciplines, la volonté d'affirmer haut et clair un Changement de perspective dans leur enseignement. Ainsi, pour Ce qui est de l'histoire, on passe de Cours où l'accent était $m$ is sur les résultats d'une recherche historique faites par les historiens eux-mêmes à des Cours qui mettent en avant les Concepts mêmes qui ont permis Cette recherche. On ajoute donc à la connaissance de savoirs factuels la maîtrise de savoirs plus démultiplicateurs, tels le nationalisme, qui donnent prise à l'analyse historique. C'est Comme Cela que j'exprimerais Ce Changement de paradigme dans l'enseignement de l'histoire mais je peux Comprendre qu'il ait été formulé, dans "l'air du temps", en termes de "CompétenCes".

\section{De $S$ tran Sfe rts p ro blé $m$ atiq Ue $S$ m ais un e piste prom ette USe Via ra catégorisation}

Un bref tour d'horizon montre le caractère essentiellement problématique des transferts, de quelque nature qu'ils soient, mais Iaisse cependant quelque espoir par le biais, préCisément, d'une Certaine catégorisation des questions proposées aux élèves.

Les recherches en psychologie Cognitive n'autorisent guère d'optimisme en matière de transfert, $p$ as plus en Ce qui Concerne les transferts d'une activité à l'autre au sein d'une même discipline que pour Ce qui est des transferts d'une discipline à l'autre. C'est Ce que souligne B. Rey (1996) avant de mettre en évidence la part d'illusion dont le concept de Compétence transversale est porteur, ConCept qu'il remplace par Celui d'intention transversale pour sou. ligner que la transversalité est plutôt de l'ordre de l'intention et du sens que possède pour un individu donné la situation nouvelle.

En Ce qui Concerne la Compétence de résolution de problèmes, le Cham d'investigation de la psyChologie Cognitive est large : Cela va des stratégies de résolution de problèmes et de la métacognition à l'étude des conditions qui facilitent ou entravent le transfert des apprentissages d'un problème à un 
autre. En particulier, Ce Courant de recherches s'intéresse aux différentes $p h$ ases de résolution de problèmes. Ainsi, A. H. Schœnfeld (1989) distingue six étapes importantes dans la résolution d'un problème, qu'il soit $m$ athém atique ou non : la lecture de l'énoncé, l'analyse du problème, l'exploration des solutions possibles, Ia planific aon d'une ou de plusieurs stratégies de solution, l'applic aon de la ou des solutions, la vérific aon de la solution en regard des données initiales. Mais, au delà de la description de Ces phases dans la résolution de problèmes ou du relevé de stratégies de résolution, je voudrais pointer iCi les recherches qui mettent en évidence un Comportement sensiblement différent entre les experts et les novices Confrontés à un problème qui relève de la Compétence des premiers. A. H. Schœnfeld (1989) observe que les uns et les autres exploitent différemment les étapes mentionnées plus haut: par exemple, les experts passent beaucoup plus de temps que les novices à analyser les données du problème. Cette observation, est à rapprocher d'une autre faite par plusieurs Chercheurs (Larkin et al. 1980 \& Chi et al., 1982) à propos de problèmes de physique : les experts passent Ce temps à situer le problème dans une classe bien identifiée, en se référant à une organisation fortement hiérarchisée de classes de problèmes qu'ils ont en mémoire. Et, il ne $m e$ parait $p$ as inutile d'y adjoindre une remarque faite par M. Crahay (2006) sur le Comportement de Ce qu'on appelle, dans Certaines entreprises, le Crisis Manager: "au fur et à mesure qu'il gère des Crises, le Crisis Manager Construit un savoir-faire d'expérience par lequel il se dote d'une classification des Crises ainsi que d'un répertoire de procédures adaptées. Bref, au fur et à mesure que le Crisis Manager acquiert de l'expertise, Ia notion de Crise se dissout progressivement".

Ces expériences soulèvent un débat sur l'opportunité de fonder un apprentissage à la résolution de problèmes sur les stratégies générales plutôt que sur les stratégies spécifiques. On identifie facilement les secondes en mathém atiques: $p a r$ exemple, la méthode de programmation linéaire ou Celle des dérivées pour optimiser une grandeur variable. Les stratégies générales sont, elles, indépendantes des Contenus disciplinaires. II s'agit, par exemple, du Chaînage arrière, au sens de E. D. Gagné (1985), qui Consiste à Considérer d'abord, non l'état initial $d u$ problème Comme dans le chaînage avant, mais le but désiré pour réduire progressivement l'écart entre ce but et l'état initial, ou encore le raisonnement par analogie sous-tendu par la question: "quel problème similaire ai-je déjà résolu ?
Notons que Cette question nous ramène aux stratégies spéCifiques. Après avoir fait écho de ce débat sensible au sein de la psychologie Cognitive, J. Tardif (1992 \& 1999) souligne l'inefficacité des enseignements de stratégies générales, telle qu'éprouvée par plusieurs recherches dont il rapporte ainsi la Conclusion : "l'enseignement de stratégies spéCifiques de résolution de problèmes est une orientation qui rend le plus probable le transfert des apprentissages ". Il clôture sa réflexion sur la résolution de problèmes et le transfert $p$ ar une synthèse relative aux facteurs influant sur l'enseignement et l'apprentissage des stratégies de résolution de problèmes. Les deux premiers facteurs sont le développement d'une base de stratégies spécifiques et l'organisation de Ces Connaissances dans la mémoire à long terme et le troisième a trait à la métacognition, à savoir l'importance d'un enseignement explicite des stratégies spécifiques et de leurs Conditions d'utilisation.

Du Côté de la didactique des mathématiques, R. Noirfalise (1991) m ise également sur des Connaissances spécifiques plutôt que sur de prétendues capacités générales, se référant entre autres aux travaux de J. Pitrat (1986) qui montrent l'importance des Connaissances (stratégies particulières) dans le jeu d'échecs. Il redéfinit le savoir procédural Comme un savoir assurant une Correspondance entre une classe de problèmes et Ce que $P$. Meirieu (1990) appelle des programmes de traitement : "Pour un apprenant, Ia classe de problèmes n'est définie que quand il a appréhendé un programme de traitement qui peut lui être appliqué; le programme de traitement, de son Côté, n'est vraiment maîtrisé que quand il a appréhendé la classe de problèmes à Iaquelle il s'applique ". Ainsi donc, apparaît un Certain Consensus sur l'importance de la notion de classes de problèmes dans la problém atique du transfert. J'ajouterais, et je le développe ailleurs (Schneider, à paraître), que ce Concept de classes de problèmes se prête particulièrement bien à l'artiCulation des trois phases importantes que M. Crahay (2006) dégage d'une synthèse diversifiée des sciences de l'éducation sur les dispositifs didactiques propres à favoriser le transfert : "Bref, pour assurer la mobilisation des connaissances en des Contextes diversifiés, il faut sans doute articuler trois moments didactiques: d'abord, une phase de Construction des apprentissages en Contexte; ensuite, une phase de déContextualisation ou de transfert (ou encore de diversification Contextuelle) et, enfin, une phase de retour réflexif ou métaCognitif sur Ces apprentissages". 
Ces quelques éléments suggèrent l'intérêt d'une catégorisation des questions étudiées à l'éCole, par le biais de savoirs spécifiques qui permettent de traiter des classes de problèmes. Mais n'est-on pas renvoyé là, du moins en Ce qui Concerne les mathé$\mathrm{m}$ atiques, à la théorie des situations didactiques de G. Brousseau (1986 \& 1998) et sa modélisation de l'apprentissage mathématique par le biais d'un jeu de situations fondamentales ? Ou bien à la théorie anthropologique du didactique de Y. Chevallard (1985 \& 1992) pour qui l'activité mathématique se décline en praxéologies : ensembles structurés de types de tâches, techniques permettant de réaliser Ces tâches et technologies et/ou théories justifiant l'efficacité d'une technique en regard d'une tâche donnée. Ce n'est pas pour rien sans doute que la théorie des situations didactiques est souvent Citée à propos des Compétences et du transfert. Mais l'est-elle à-propos ? Dans un autre article (Schneider, à $p$ araître), je développe, d'une part, que Ces références sont peu étayées et révèlent une forte influence de l'idéologie ambiante sans réelle problé$m$ atisation et, d'autre part, que les deux théories didactiques Citées supra permettent de penser efficacement le problème $d u$ transfert, alors même que Ce Concept n'y est pas repris de manière explicite, au point de pouvoir suggérer des éléments d'interprétation pour d'autres disciplines.

\section{EN GUISE DE CONCLUSION : LA NÉCESSITÉ D. UNE DIMENSION ÉPISTÉMOLOGIQUE}

Je voudrais, pour Conclure, insister sur l'importance d'une dimension épistémologique dont la mouvance des Compétences fait l'éConomie, me sem ble-t-il.

Je le ferai d'abord en revenant à l'espoir de transversalité qui semble légitimer la formulation des objectifs de l'enseignement en termes de Compétences transversales. Il semble qu'on mésestime là le fait que l'exercice dans une discipline d'une Compétence "candidate " à la transversalité ne favorise pas forCément son exerCice dans une autre discipline tant sont différents et spéCifiques les "espaces didactiques" (au sens de Leutenegger et al., 2003) que Constituent les multiples disciplines abordées par un élève lors de sa scolarité, mobilisant des approches, des normes, des Codes... particuliers, non réductibles les uns aux autres sauf à sacrifier l'épistémologie propre de Ces différentes disciplines et risquer ainsi de favoriser des appren- tissages "Creux" Chez les élèves au sein de ChaCune d'elles. J'illustre (Schneider, 2004), par exemple, les formes très spéCifiques que prennent, au sein des mathém atiques, des Compétences transversales telles que: faire preuve d'esprit Critique, formuler et valider des hypothèses, Communiquer, montrant par là que les intentions rationnelle et scripturale telles que définies par B. Rey (1996) supposent, en mathématiques, une m aîtrise profonde de Cette disCipline et de ses ressorts épistémologiques. Aussi néglige-t-on sans doute, derrière la recherche d'un discours Commun, les obstacles que doit franChir un même élève pour se situer efficacement dans Chacun de ces "espaces didactiques" alors que praticiens et Chercheurs attestent de la difficulté de passer de l'un à l'autre (Leutenegger et al., 2003).

Mais le choix même des savoirs disciplinaires relève d'une analyse épistémologique de la discipline, des pratiques quilui sont liées dans une institution donnée et d'une étude de sa transposition didactique - je renvoie ici à la note de synthèse de A. MerCier (2002). Une réflexion de Ce type Conduit bien vite à une grande différenciation disciplinaire. Ainsi, Comme le montre M. Caillot (1996), des dis Ciplines Comme la physique et Ia Chimie, d'habitude perçues assez proches, se distinguent fortement par leur rapport au savoir: "Chez les physiciens l'intelligibilité du monde physique et de l'univers est primordiale, ce qui place la recherche scientifique au Cœur de la production des Connaissances en physique, la technologie n'est alors vue que Comme la $m$ ise en forme, l'application d'un savoir élaboré par ailleurs. Physique et technologie sont alors Considérées Comme deux dom aines différents. Par Contre, pour le Chimiste, la production de Connaissances scientifiques est inséparable de la technologie puisque son but est de transformer la matière, Ce qui place sur un pied d'égalité recherche scientifique et recherChe teChnologique». À Cela s'ajoute "Ia place de la Chimie dans l'éConomie et la société (Caillot, 1996). Il existe une industrie Chimique forte et puissante qui n'a pas sa contrepartie en physique. "Plus généralement, M. Caillot développe que le rapport entre savoirs savants et savoirs enseignés, au Cœur $d u$ phénomène $d e$ transposition didactique, se décline fort différemment en français, en histoire et géographie ou encore dans des disCiplines plus liées à des pratiques professionnelles.

Ces analyses renvoient à la catégorisation des questions et donc à leur "disciplinarisation", ainsi qu'aux paradigmes et méthodes qui ont été au prinCipe de Cette catégorisation. Par exemple, Comme 
développé dans l'EnCyClopædia Universalis (1980), l'émergence de l'histoire en tant que discipline scientifique s'est ac $6 \mathrm{mpagnée}$ d'une spécific aon des questions étudiées et des méthodes associées qui relèvent tantôt de l'histoire géographique, de I'histoire économique ou de Celle des mentalités. Les Concepts eux-mêmes autorisent une classific aon plus fine, ainsi qu'illustré par le cours d'A. Maingain (2000), Cité supra. Et, au niveau plus b gique de l'étude du milieu, on peut bien imaginer l'intérêt de distinguer, par exemple, les milieux ruraux des milieux urb ens, en Ce sens qu'ils relèvent sans doute d'invariants distinCts. L'analyse de la transposition didactique, engagée depuis longtemps en $m$ athématiques, est encore b 由utiante dans d'autres disciplines. Je la vois poindre dans ces propos de didacticiens belges de l'histoire et de la formation historique : "La question des savoirs à enseigner mériterait également d'être (re)posée. La finalité ultime du cours d'histoire se marie-t-elle harmonieusement avec la persistance d'un $c$ are de Contenus-matières que d'aucuns jugeront clas- siques. Le Choix d'inscrire, au cœur de la nouvelle approche, l'apprentissage de Concepts - et de Ces Concepts-là - est-il pertinent ? Sur le plan plus strictement didactique, Comment Ce travail de Conceptualisation progressive va-t-il se moduler, dans le Concret des classes? De quel outillage Conceptuel l'élève term inant ses hum anités générales disposerat-il effeCtivement? " (Jadoulle \& Bouhon, 2003). Ces questions signent, parmi d'autres, une réflexion proprement didactique et on peut se demander pourquoi leurs auteurs CherChent à se disculper de les avoir posées.

Ces quelques exemples illustrent, par Contraste, l'éCrasement épistémologique dont est porteur un Certain discours sur les Compétences et sur Ia transversalité. Ils montrent l'ampleur du travail qui doit être poursuivi dans chacune des disciplines et qu'il ne m'appartient pas de mener ici...

Magg y Schneider-Gilot maggy.schneider@iundp.ac.be Facultés universitaires de Namur

\section{BIBLIOGRAPHIE}

BeCkeRs J. (2002). Développer et évaluer des Compétences à l'école: vers plus d'efficacité et d'équité. Bruxelles : Labor.

Belgique : communauté fRANÇAISE (1997). 24 juillet 1997 : déCret définissant les $m$ issions prioritaires de l'enseignement fondamental et de l'enseignement seCondaire et organisant les structures propres à les atteindre, $n \circ 1997-07-24 / 51$.

Brousseau G. (1986). "Fondements et méthodes de la did actique des m athém atiques". ReCherches en didactique des mathém atiques, vol. 7, no 2, p. 33-115.

Brousseau G. (1998). La théorie des situations didactiques. Grenoble : Éd. La Pensée sauvage.

Calleot M. (1996). "La théorie de Ia transposition didactique est-elle transposable? "In C. Raisky \& M. Caillot (éd.), Au-delà des didactiques, le didactique. Bruxelles: De Bo eck, p. 19-35.

Cazzaro J. - P. ; Noël G. ; Pourbaix F. \& Tilleuil P. (2001). StruCturer l'enseignement des $m$ athém atiques par des problèmes. Bruxelles: De Boeck.

Cecafoc (2003). Outils d'évaluation en histoire. Bruxelles: Fédération de l'enseignement secondaire catholique [document interne].

Chevallard Y. (1985). La transposition didactigue : du savo ir savant au savoir enseigné. Grenoble: Éd. La Pensée Sauvage.
Chevallard Y. (1992). "Concepts fondamentaux de la didactique : perspectives apportées par une approche anthropologique". Recherches en didactique des $m$ athém atiques, vol. $12, n^{\circ} 1, \mathrm{p} .72-112$.

Chevallard Y. \& Matheron Y. (2002). "Travaux personnels encadrés : un cadre d'analyse didactique pour un Changement majeur dans l'enseignement au LyCée". In Nouveaux dispositifs d'enseignement en mathématiques dans les Collèges et les lyCées. Actes des journées de Dijon: 24-25 m ai 20 0.2Dijon : IREM de Diion, p. $141-150$.

Chi M. T. H. ; Glaser R. \& Rees E. (1982). "Expertise in problem solving". In R. J. Sternberg (dir.), Advances in the psychology of hum an intelligence. Hillsdale [NJ]: L. Erıbaum, p. 161-183.

Crahay M. (2006). "Dangers, in Certitudes et in Complétude de la logique de la Compétence en éducation". Revue française de pédagogie, n०154, p. 97-110.

Draelants H. ; Dupriez V. \& Maroy C. (2003). Le système scolaire [Dossier n ${ }^{\circ} 59$ ]. Bruxelles: Centre de recherche et d'inform ation socio-politiques [CRISP].

EnCyClop EDIA UnIVERSALIS (1980). Histoire de l'histoire. Paris : En Cy Clopædia Univers alis, vol. 8.

FESEC (2000). Program me $n^{\circ} D / 2000 / 73627 /$ d'histoire et de formation historique des $2^{e}$ et $3^{e}$ degrés des humanités générales et teChnologiques. Bruxelles: Fédération de l'enseignement second aire Catholique. 
FoureZ G. (1998). La ConstruCtion des sciences. Bruxelles: De Bo eck.

FouREZ G. (2003). Apprivoiser l'épistémologie. Bruxelles : De Bo e Ck.

GaGne E. D. (1985). The Cognitive psyChology of school learning. Boston: Little, Brown and Company.

Gillet P. [éd.] (1991). Construire des form ations. Paris : ESF.

Jadoulle J.-L. \& Bouhon M. (2003). Développer des Compétences en classe d'histoire. Louvain : université catho. lique de Louvain : unité de didactique et de Communication en histoire.

JoHSUA S. (2002). "La popularité pédagogique de la notion de Compétence peut-elle se Comprendre Comme une réponse inadaptée à une difficulté didactique majeure ? ". In J. Dolz \& E. Olıagnier (éd.), L'énigme de la Compétence en éducation. Bruxelles: De Boeck, p. $115-128$

Jonnaert P. (2003). Compétences et so Cio ConstruCtivisme. Bruxelles: De Boeck.

LARKIN J. H. ; McDermott J.; Simon D. P. \& Simon H. A $(1980)$. "Expert and novice perform ance in solving physics problems". SCienCe, vol. 208,p.1335-1442.

LEPLAT J. (1992). "L'analyse psychologique du travail ". In J. Leplat (Coord.) L'an alyse du travail. Tou louse : Octarès.

Leutenegger F. ; Schubauer M.-L. \& Amade- Escot C. (2003). Recherche et intervention didactique : quelle articula tion entre les dispositifs de recherche et les dispositifs de formation àl'action enseignante? Texte de cadrage du Symposium $n^{\circ} 9 \mathrm{~d} u$ Réseau éducation formation (REF). Université de Genève : Faculté de psychologie et de sCiences de l'éducation, 18-19 septembre 2003.

Maingain A. (2000). Cours d'histoire destiné àdes élèves en fin de CyCle seCondaire. Bruxelles: Collège SaintMichel [publication interne].

Maingain A.; Dufour B. \& Fourez G. (2002). Approches did actiques de l'interdis Cip lin arité. Bruxelles: De Boeck.

MATHERON Y. \& SCHNEIDER M. (2005). "L'interdis Cip lin arité à l'étude". In A. Mercier \& C. Margolinas (éd.), Balises en didactique des mathém atiques: aces de la X qlécole d'été de didactique des mathém atiques: Corps, aût 20 o. Grenoble : Éd. La pensée sauvage [CD-Rom ]

MERCIER A. (2002). "Latransposition des objets d'enseignement et la définition de l'espace didactique, en mathématiques". Revue française de pédagogie, n 141 , p. $135-172$.

Meirieu P. (1990). "Guide pour la pratique du Conseil méthodologique". Cahiers pédagogiques, n²84-285, p. $61-67$.

NoIrfalise R. (1991). "Connaissances ou capacités?". Repères-IREM, $\mathrm{n}^{\circ} 5$, p. 5-22

Pallascio R. (2000). "Vers une Contextualisation des apprentissages par CompétenCes ". [DoCument éleCtronique définitivem ent indisponible]

Perkins D. \& Salomon G. (1989). "Are Cognitive Skills Context-Bound?"EduC aonal ResearCher, vol. 17, p. $16-25$.
Perrenoud P. (2002). "D'une m étap hore à l'autre : transférer ou mobiliser ses Connaissances ? ». In J. Dolz \& E. Ollagnier (éd.), L'énigme de la Compétence en eduC aon. Bruxelles: De Boeck, p. 45-60.

Pitrat J. (1986). "Connaissances el métaconnaissances". In J.-L. Lemoigne (éd.), IntelligenCe des méc aismes et $m$ éc aisme des intelligenCes. Paris: Fayard, p. 73-113

Pólya G. (1967). La déCouverte des mathém atiques. Paris: Dunod.

QuÉBEC : Ministère de L'Éducation, dU LOISIR ET DU SPORT (2001). Programme de formation de l'éCole québéCoise: éduC aon préscolaire, enseignement prim aire. Québec: MELS. Document disponible sur Internet au form at PDF à l'adresse : http://www.mels.gouv.qC.ca/DGFJ/dp/ programme_de_form ation/prim aire/pef/prfrom $2001 / \mathrm{prf}$ orm 2001 .pdf (Consulté le 6 février 2006 ).

Raynal F. \& Rieunier A. (1997). Pédagogie: dictionnaire des ConCepts Clés. Apprentissage, form ation et psyChologie Cognitive. Is sy-les-Mouline aux : ESF.

REY B. (1996). Les Compétences transversales en question. Paris: ESF.

Rey B. ; Carette V. \& Kahn S. (2002). Lignes direCtrices pour la Construction d'outils d'évaluation relatifs aux socles de CompétenCes. Rapport de reCherChe. Bruxelles: université libre de Bruxelles : service des sciences de l'éducation.

SCHNEIDER M. (2002 a). "Problè $\mathrm{m}$ es et situations-problè $\mathrm{m}$ es : un regard pluraliste". Mathématique et pédagogie, $n \circ 137, p .13-48$.

SCHNEIDER M. (2002b). "À propos de l'évaluation des Compétences en mathématiques: le cas de la résolution de problèmes". In GRIFED (éd.), L'évaluation des CompétenCes Chez l'apprenant. Louvain-la-Neuve : Presses universitaires de Louvain, p. 37-45.

SCHNEIDER M. (2004). "Viser le "transversal " à travers du "bon disciplinaire" ou tro is Compétences transversales Contextualisées au sein de l'enseignement des $\mathrm{m}$ athém atiques ". Repères-IREM, n०55, p. 51-70.

SCHNEIDER M. (à paraître). "Comment des théories didactiques permettent-elles de penser le transferten $m$ athématiques ou dans d'autres disciplines? "ReCherches en didactique des mathém atiques [accepté pour publication ]

Schoenfeld A. H. (1989). "Teaching $m$ athem atical thinking and problem solving". In L. B. Resnick \& L. E. Klopfer (dir.), Tow ard the thinking CurriCulum : Current Cognitive researCh. Alexandria: Association for Supervision and Curriculum Development, p. 83-104

SFARD A. (1991). "On the dual nature of mathematical Conceptions: reflections on processes and objeCts as different sides of the same Coin ". EduC aonal Studies in Mathem atics, vol. 22,p. 1-36

TARDIF J. (1992). Pour un enseignement stratégique. Montréal : Éd. Logiques.

TARDIF J. (1999). Le transfert des apprentissages. Montréal: Éd. Logiques. 